\title{
Data Space Randomization (DSR)
}

Sandeep Bhatkar

Symantec Research Labs

\author{
R. Sekar \\ Department of Computer Science \\ Stony Brook University
}

Detection of Intrusions and Malware \&Vulnerabilities Assessment

July 10, 2008

Paris, France 


\section{Importance of Memory Error Exploits}

- Memory error exploits continue to be the dominant threat

- Behind most "critical updates" from Microsoft and other vendors

- Mechanism of choice in "mass market" attacks, including worms

- Defense techniques to address this problem continues to be the hot topic of research

- Over 20 techniques have been invented so far

- Techniques that provide full protection haven't been practical

- High performance cost

- Code compatibility issues

- Diversity based defenses emerging as more promising

- Address Space Randomization (ASR)

- Instruction Set Randomization (ISR) 


\section{Previous Diversity Based Techniques}

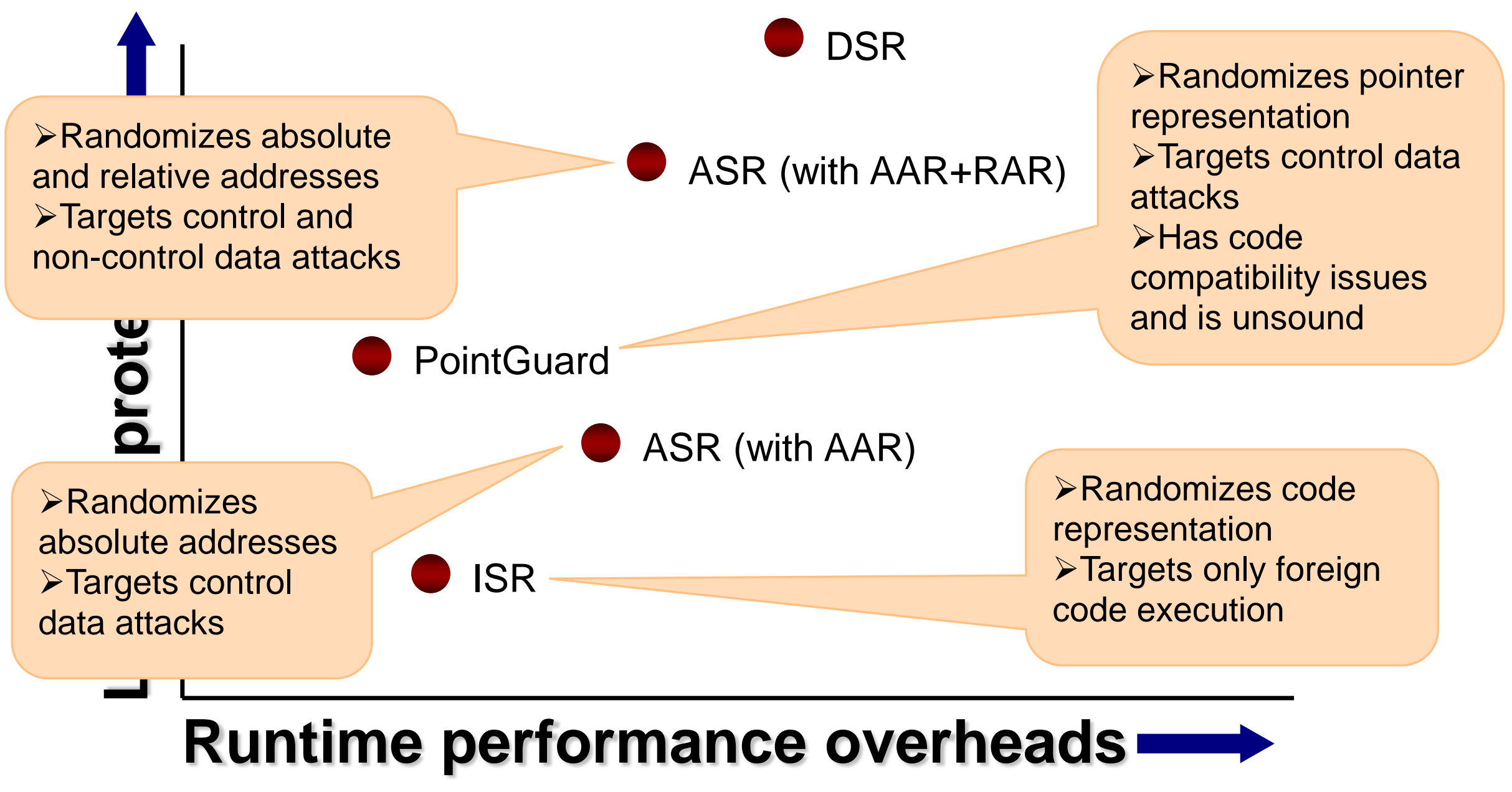

RAR: Relative Address Randomization

AAR: Absolute Address Randomization 


\section{DSR Technique}

- Basic idea: randomize data representation

- Xor each data object with a distinct random mask

- Effect of data corruption becomes non-deterministic

- Example: use out-of-bounds access on array $\boldsymbol{X} 1$ to corrupt variable $X 2$ with value $V$

- Actual value written: $\operatorname{mask}(\boldsymbol{X} 1) \oplus \boldsymbol{V}$

- When $\boldsymbol{X} \mathbf{2}$ is read, its value interpreted as $\operatorname{mask}(\boldsymbol{X} \mathbf{2}) \oplus(\operatorname{mask}(\boldsymbol{X} \mathbf{1}) \oplus \boldsymbol{V})$

$-\operatorname{mask}(\boldsymbol{X} 2) \oplus \operatorname{mask}(\boldsymbol{X} \mathbf{1}) \oplus \boldsymbol{V} \neq \boldsymbol{V} \quad(\operatorname{because} \operatorname{mask}(\boldsymbol{X} \mathbf{2}) \neq \operatorname{mask}(\boldsymbol{X} \mathbf{1}))$

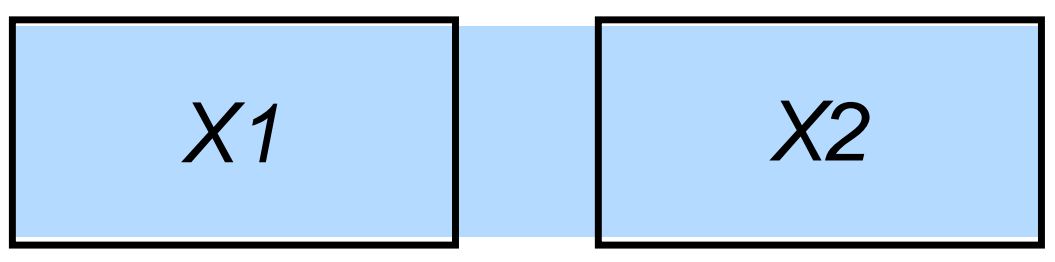

Example: Buffer overflow 


\section{Differences with PointGuard}

- DSR randomizes all data objects, not just pointers

- PointGuard breaks working programs, DSR doesn't

- Attacks targeted:

- PointGuard targets absolute address-dependent attacks (pointer corruption)

- DSR targets relative address-dependent attacks

- Helps defeating non-control data attacks that corrupt files names, userids, command names, authentication data, ...

- Automatically defeats absolute address-dependent attacks as pointer corruption step is relative address-dependant

- Unlike PointGuard, DSR is not vulnerable to information leakage attacks (details forthcoming) 


\section{DSR Transformation Approach}

- For each variable $\boldsymbol{v}$, introduce another variable $\boldsymbol{m} \_\boldsymbol{v}$ for storing its mask

- Randomize values assigned to variables (LHS)

- Example: $x=5 \Rightarrow x=5 ; x=x^{\wedge} m_{-} x$;

- Derandomize used variables (RHS)

- Example: $(x+y) \Rightarrow\left(\left(x^{\wedge} m_{-} x\right)+\left(y^{\wedge} m_{-} y\right)\right)$

- Key problem: aliasing

int $x, y,{ }^{*} p t r ; \ldots$

ptr $=\& x ; \ldots$

$p t r=\& y ; \ldots$

$z={ }^{*} p t r$

- Unfortunately, we cannot statically determine the mask associated with * $p t r$ - it could be that of $x$ or $y$ 


\section{Aliasing Problem}

- Solution to aliasing problem: assign the same randomization mask to possibly aliased objects

- Requires alias analysis

- Current implementation supports Steensgaard's algorithm for alias analysis

- Flow-insensitive

- Context-insensitive

- Field-insensitive

- All heap objects allocated at the same point represented by a single logical object

- Linear time complexity 


\section{Pointer Analysis \& Mask Assignment}

int intval;

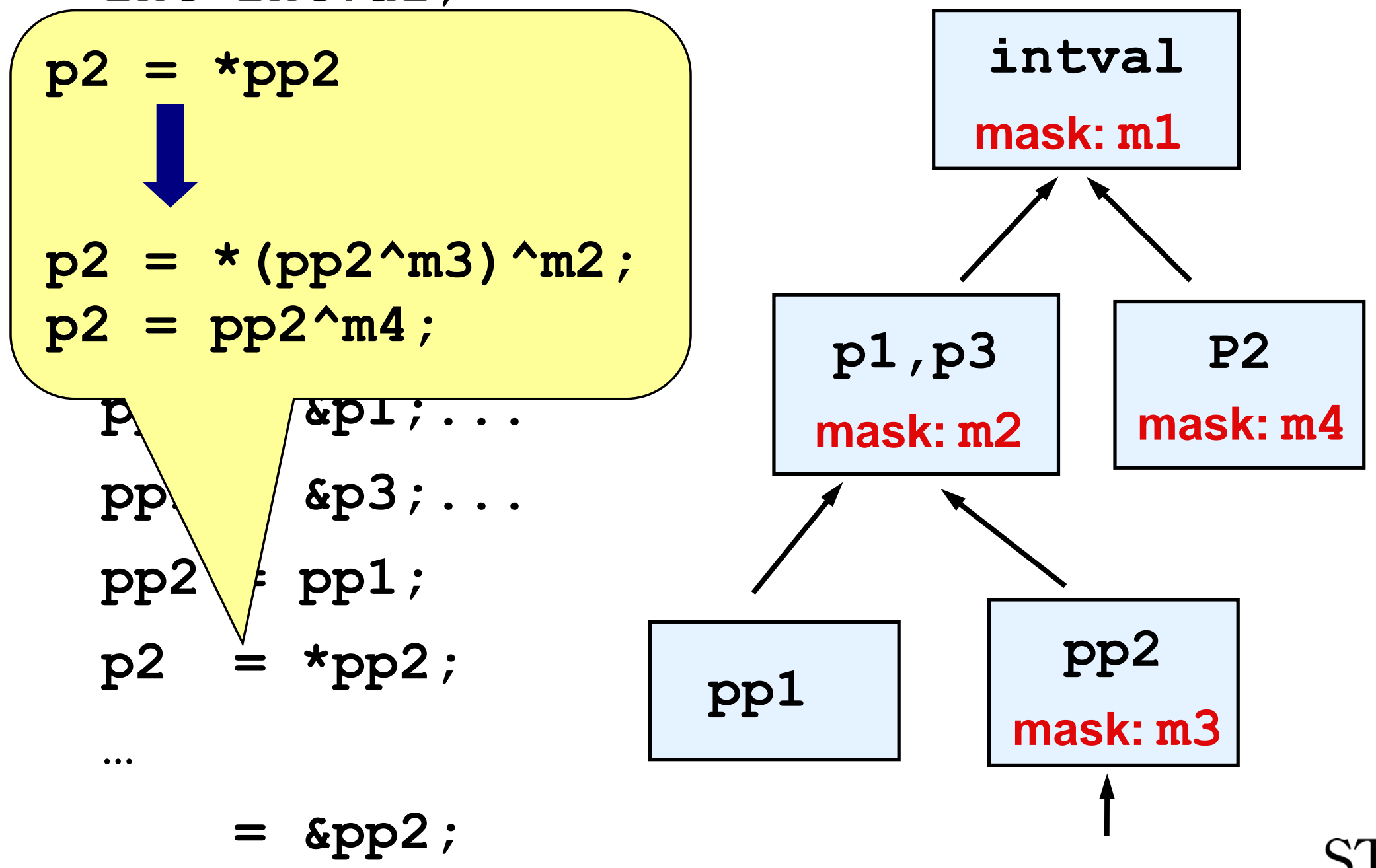




\section{Optimization}

- Basic idea: mask only overflow candidate objects (OCOs),

e.g., arrays, structures containing arrays, objects whose addresses are taken

- Optimization is very effective because majority of memory access in a typical program are to non-OCOs

- Ensure that optimization doesn't significantly impact security

- Claim: all data corruptions involve overflows from OCOs

- All relative address-dependent attacks involve overflows from OCOs

- All absolute address-dependent attacks involve corruption of pointers

- Require a relative address-dependent step, e.g., buffer overflow, integer overflow, heap overflow, etc.

- Implication: need protection from overflows in OCOs 


\section{Protection from Overflows in OCOs (Optimization ctd)}

- Protect non-OCOs from overflows in OCOs

- Non-OCOs separated from OCOs with an unmapped memory page

- Guard against overflows among OCOs

- Use of distinct masks provides automatic protection for overflows between unaliased OCOs

- Prevent overflows between aliased OCOs by allocating them in disjoint memory regions

- Stack: allocate local OCOs on disjoint stacks (buffer stacks) if small in number; allocate in heap if the number is high

- Static: number of disjoint memory areas statically known

- Heap: heap OCOs allocations (typically large in number) randomly distributed in a fixed number of heap memory regions 


\section{Implementation}

- Based on source-to-source transformation of C programs

- Uses CIL as front-end and OCAML as implementation language

- Implementation issues

- Handling overflows within structures

- Use field-sensitive pointer analysis so as to assign distinct mask to each field of a structure (not done yet)

- Handle functions such as memcpy, bzero in a context-sensitive way

- Handling variable argument functions

- Treat them as if they take array (with maximum size limit) parameter

- Transformation of libraries

- Source code available: need dynamic mask resolution

- Source code unavailable: need summary functions for library calls 


\section{Execution Time Overheads}

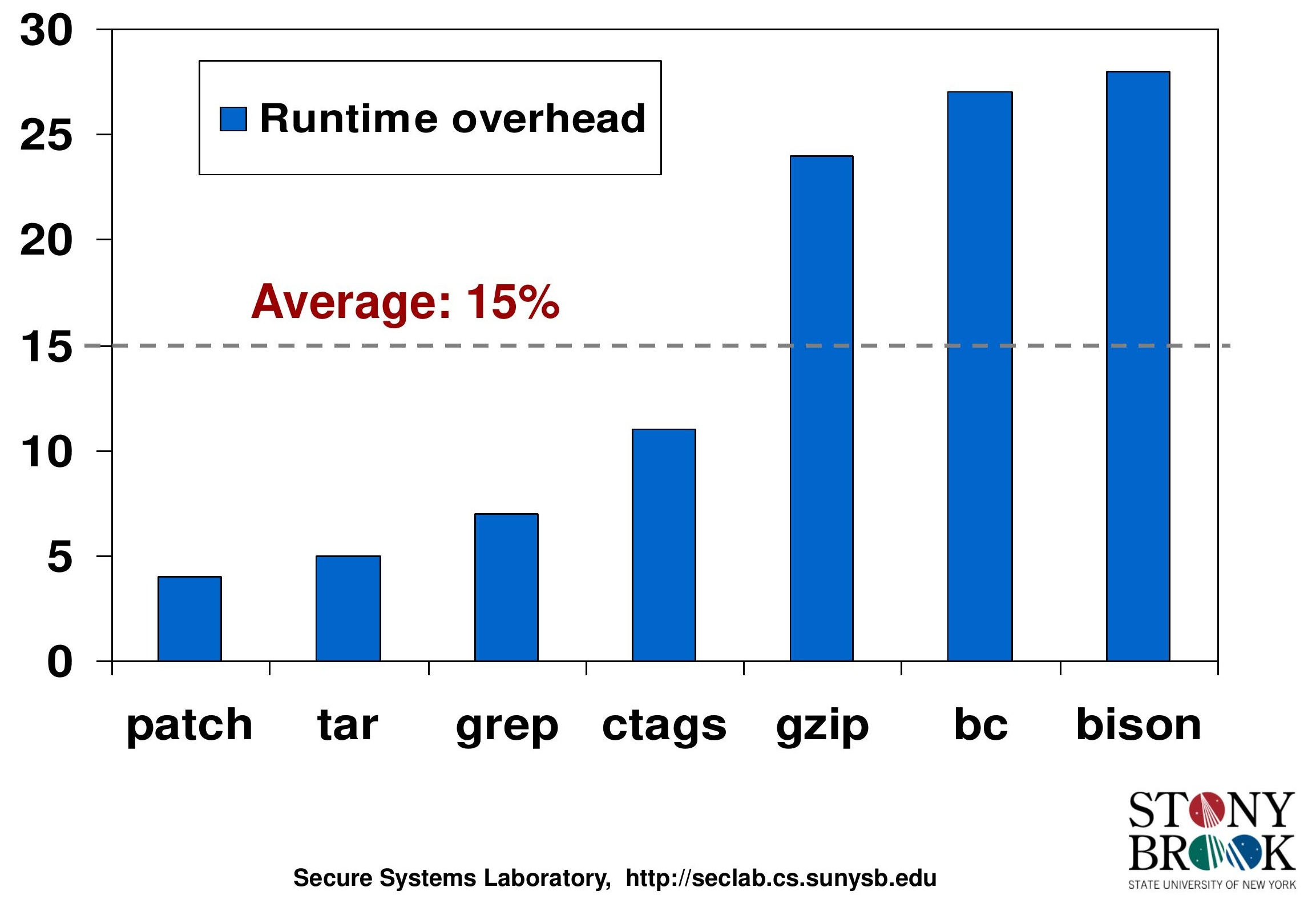




\section{Effectiveness Against Various Attacks}

- Stack buffer overflows

- Overflows to corrupt data on main stack (e.g., return address, based pointer, saved registers) fail

- Overflows among overflow candidate objects

- fail if source and target objects are in different buffer stack or disjoint memory regions

- succeed with probability $2^{-32}$ otherwise

- Static buffer overflows

- Overflows to corrupt non overflow candidate objects fail

- Overflows between overflow candidate objects

- fail if source and target objects are in different memory regions

- succeed with probability $2^{-32}$ otherwise 


\section{Effectiveness Against Various Attacks}

- Heap overflows

- Traditional attack (corruption of heap control data) succeeds with probability $2^{-32}$

- An overflow from one heap block to the next succeeds with probability $>2^{-32}$ (property of a program)

- Heap objects randomly distributed

- Nonetheless, such overflows also detected when control data between the heap blocks get corrupted

- Format string attacks

- Traditional attack with on directive fails

- DSR cannot stop attacks that print contents of stack with $\frac{0}{x}$

- Relative address attacks based on integer overflows

- If source and target objects share the same mask, such attacks can be successful (protection provided in the form of RAR) 


\section{Effectiveness Against Attacks targeting DSR}

- Information leakage attacks

- If a masked data is leaked, an attacker can deduce the mask if the plaintext data value is known

- Attempt to read masked data results in reading plaintext data

- Brute force and guessing attacks

- become difficult because of low probability of success

- Partial pointer overwrites

- become impossible on stack-resident data because the main stack does not contain overflow candidate objects 


\section{Related Work}

- Runtime guarding: StackGuard, StackShield, RAD, Libsafe, Libverify, ProPolice, FormatGuard, ...

- Attack specific, no comprehensive protection

- Runtime bounds and pointer checking: [Austin+94],

[Jones+97], Cyclone, CCured, [Ruwase+04], [Xu+04],

[Dhurjati et al 06]

- High overheads or incompatibility with legacy code

- Runtime enforcement of static analysis results: CFI, DFI, WIT

- Don't target all exploits (e.g., data leakage/corruption)

- Randomization techniques: ASR (PaX, [Bhatkar+03], [Xu+03]), ISR ([Barrantes+03], [Kc+03]), PointGuard

- No or limited protection from non-control data attacks 


\section{Summary of Contributions}

- Randomization of all types of data provides comprehensive coverage

- Control data attacks

- Non-control data attacks

- Unlike other randomization techniques, resistant to information leakage attacks

- Higher range of randomization than other randomization techniques

- Capable of detecting exploits that are missed by full boundschecking techniques

- Example: overflows within structures

- Low runtime overhead

- Average around $15 \%$ 


\title{
Thank You!
}

\author{
R.Sekar \\ Email:sekar@cs.sunysb.edu
}

